\title{
The Effect of Out-of-Plane Strain on the Electronic Properties of Zigzag Graphene Nanoribbons
}

\author{
Xiaohui Hu*, Xiao Xie, Litao Sun \\ *SEU-FEI Nano-Pico Center, Key Laboratory of MEMS of Ministry of Education, Southeast University, 210096 Nanjing, China \\ *Corresponding Author: Litao Sun, slt@seu.edu.cn
}

\begin{abstract}
We have studied the influence of out-of-plane strain on the electronic properties of zigzag graphene nanoribbons (ZGNRs) using first principles methods. We find that the electronic properties of ZGNRs are highly sensitive to the strains. As the strain increases, the band gap first remains almost invariant and then decreases monotonically, which could be used to design high precision strain sensors. ZGNRs based sensors would possess higher sensitivity and quicker response compared to the conventional sensors.
\end{abstract}

Keywords-zigzag graphene nanoribbons; band gap; strain sensors

\section{INTRODUCTION}

Graphene, a single atomic layer of graphite, has been successfully produced in experiments [1, 2], which have resulted in intensive investigations on graphene-based structures because of fundamental physics interests and promising applications [3]. However, perfect graphene is a zero gap semimetal, which significantly limits the application of graphene in nanodevices, for example in $\mathrm{p}-\mathrm{n}$ junctions or transistors. Thus, it is necessary to develop a simple and reliable approach to tune the electronic structure of graphene into semiconductive. Several methods have been developed to tailor its electronic or magnetic properties, such as doping [4], electric field application [5] or by substrate proximity effects [6]. The interaction between transition metal atoms and vacancies in graphene has also been considered in recent experiments $[7,8]$ and simulations $[9,10]$ in the context of graphene electronic structure modification.

Graphene nanoribbons (GNRs), one dimensional structure of graphene, are more fascinating due to their tunable electronic properties depending on different edge patterns. Theoretical studies predict that zigzag GNRs (ZGNRs) have special localized magnetic edge states, which are antiferromagnetically coupled between the two edges, while armchair GNRs (AGNRs) are nonmagnetic and semiconducting $[11,12]$. The capability to control GNRs' electronic properties is highly desired to build future nanodevice directly on GNRs. The ways to modify the electronic and magnetic properties of GNRs have been extensively investigated, including chemical doping [13-15] and mechanical deformation [16-20]. Recently, several reports have examined graphene under the plane uniaxial deformation, but no significant changes in graphene's band structure have been found for reversible strains of up to $20 \%$ [21, 22]. The

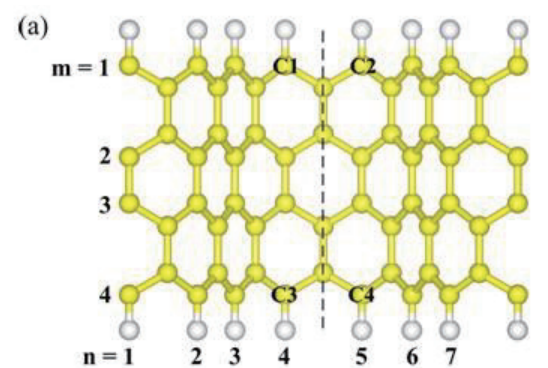

(b)



Fig. 1. (a) Top view and (b) side view of the model structural. The edge carbon atoms close to the curving sites are denoted as C1, C2 and C3, C4. The yellow balls stand for carbon atoms, and the white balls represent hydrogen atoms.

effect of the uniaxial stain on GNRs has also been studied and found that the electronic structures of ZGNRs are not sensitive to uniaxial strain, which however can modify AGNRs significantly [16]. It is great interest to know how the out-ofplane strain may modify the properties of the ZGNRs. However, information in this area is lacking.

Here we demonstrate a simple way to change electronic properties of ZGNRs by compressing flat ZGNRs along the periodic direction using first principles simulations. As the strain increases, first the band gap does not change considerably but then it decreases monotonically. The diverse electronic property of ZGNRs is hopeful to develop and fabricate high sensitive strain sensors.

\section{Method AND ModeL}

Calculations were performed using the Vienna $\mathrm{Ab}$ Initio Simulation Package (VASP) code $[23,24]$ based on DFT. The generalized gradient approximation (GGA) with the PerdewBurke-Ernzerhof (PBE) parametrization [25] was chosen for the exchange-correlation functional. The core electrons were accounted for within the projector augmented wave (PAW) approach [26, 27]. The plane wave cutoff energy was set to 
$500 \mathrm{eV}$, because a higher value had little effect on the results. The structures were relaxed until the energy and the force on each atom were less than $10^{-5} \mathrm{eV}$ and $0.01 \mathrm{eV} / \AA$, respectively. A $19 \times 1 \times 1 k$-point mesh with Monkhorst-Pack scheme was used for sampling the 1D Brillouin zone. In order to simulate isolated ribbons, the intervals among the ribbons were kept as large as $12 \AA$ for both layer-layer and edge-edge distances.

Following the previous convention [14], the ZGNRs are defined with $m \times n$ indices, where $m$ and $n$ are the number of rows and columns across the ZGNR width and length, respectively. Here we choose the ZGNR of $m=4$ and $n=7$ as the typical system, as shown in Fig. 1(a). The edge carbon atoms are all saturated by hydrogen atoms to avoid dangling bonds. The model structure is constructed by symmetrically bending the ribbon from the middle of the supercell (dash line in Fig. 1(a)) along the periodic direction. The edge carbon atoms close to the curving sites are indicated by $\mathrm{C} 1, \mathrm{C} 2$ and C3, C4. The deformation of ZGNRs can be represented by the strain $\eta$, defined as $\eta=\left(r_{0}-r\right) / r_{0}$, where $r_{0}(17.22 \AA)$ and $r$ are the flat and deformed equilibrium lattice constants along the periodic direction of ZGNRs, as denoted in Fig. 1(b). A series of out-of plane strains are investigated. For simplicity, the deformed ZGNRs are named as $\eta$-ZGNR in this study. The positions of all carbon and hydrogen atoms in the supercell are not constrained and could be fully relaxed under the condition that the cell parameters are fixed to the values optimized for the original supercell. After full relaxation, the geometric structures demonstrate periodic, wavelike geometries, as illustrated in Fig. 1(b).

\section{RESULTS AND DisCUSSION}

To investigate the effect of out-of-plane strain on the electronic properties of ZGNR, the lowest-energy state of the deformed ZGNR needs to be calculated. Previous theoretical studies have revealed that flat ZGNR has an antiferromagnetic ground state $[12,28]$. To determine the ground state of the deformed ZGNR, we perform the spin-polarized total energy calculations for different magnetic phases of a series of deformed ZGNRs, including nonmagnetic (NM),

TABLE I. THE ENERGY DIFFERENCE PER SUPERCELL BETWEEN THE NONMAGNETIC STATE (NM) AND THE MAGNETIC STATE (BOTH AFM AND FM) AT THE DIFFERENT STRAIN

\begin{tabular}{|c|c|c|}
\hline $\boldsymbol{\eta}(\boldsymbol{\%})$ & NM-AFM $(\mathbf{e V})$ & NM-FM $(\mathbf{e V})$ \\
\hline 0 & 0.38 & 0.28 \\
\hline 7.6 & 0.37 & 0.27 \\
\hline 13.4 & 0.38 & 0.28 \\
\hline 20.7 & 0.39 & 0.28 \\
\hline 29.9 & 0.41 & 0.30 \\
\hline 30.5 & 0 & 0 \\
\hline 35.7 & 0 & 0 \\
\hline 41.2 & 0 & 0 \\
\hline 47.0 & 0 & 0 \\
\hline 53.1 & 0 & 0 \\
\hline 56.2 & 0.08 & 0.03 \\
\hline
\end{tabular}

antiferromagnetic (AFM), and ferromagnetic (FM) ones. TABLE I shows the energy difference per supercell between the nonmagnetic state and the magnetic state (both AFM and FM) at the different strain $\eta$. We found that in the strain range of $0 \leq \eta \leq 29.9 \%$, the energy of AFM state is lower compared to the corresponding FM state, suggesting that AFM is always the ground state for the low strain. Increasing the strain, the energy difference abruptly drops to zero and the ZGNR becomes nonmagnetic, where $\eta=30.5 \%$ is the critical value which separates the magnetic state from nonmagnetic state. Further elevation does not change the nonmagnetic state until $\eta=56.2 \%$, where AFM state dominates again. Thus, we found an out-of-plane strain-induced transition from AFM to NM and then back to AFM.

What causes the magnetic transformation? It is well known that flat ZGNR has the edge states mainly localized on the two edge carbon atoms, which form the flat bands at the Fermi level and thus to give rise to a sharp density of states (DOS) [28]. In the mean-field Hubbard model [28, 29], it is believed that the peak in the DOS results in a magnetic instability, where the introduced electron-electron interaction leads to spin splitting of the edge states. The feature is also confirmed by first-principles studies [30-32].

Consequently, we expect that the magnetic transformation is relative to the DOS at the Fermi level. To answer this question, we investigate the change of DOS at the Fermi level for different strain effect in the spin-nonpolarized case. Specifically, we present the DOSs of several typical configurations for 29.9\%-ZGNR, 30.5\%-ZGNR and 56.2\%ZGNR in Fig. 2. Clearly, 29.9\%-ZGNR has a large DOS at the Fermi level, similar to that of the flat ZGNR. The sharp DOS induces spin splitting of the edge states and there will be a magnetic transition into the AFM ground state. For 30.5\%ZGNR, an interesting phenomenon appears that the DOS at the Fermi level becomes zero, which cannot result in magnetic instability, so it is nonmagnetic. However, for 56.2\%-ZGNR, there is the DOS appears at the Fermi level, which leads to the AFM ground state. The results have indeed confirmed the above mentioned mechanism.

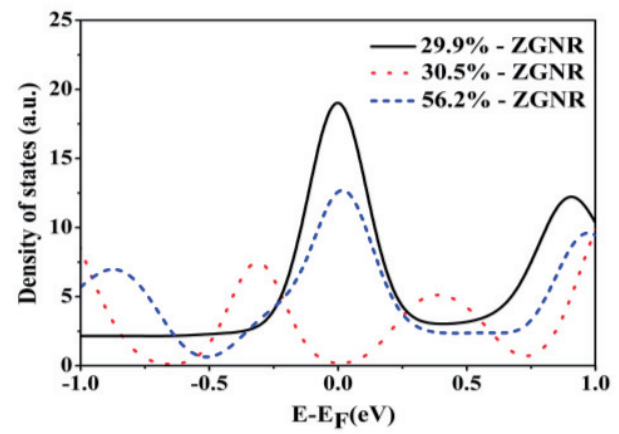

Fig. 2. The total spin-nonpolarized DOS for $29.9 \%$-ZGNR, $30.5 \%$-ZGNR and $56.2 \%$-ZGNR. Because of the presence of large DOS at the Fermi level, there will be a magnetic transition into the AFM ground state for $29.9 \%$-ZGNR and $56.2 \%$-ZGNR; while there is no DOS at the Fermi level for $30.5 \%$-ZGNR and it is nonmagnetic. 
TABLE II. THE BAND GAP OF THE ZGNR AT DIFFERENT STRAINS

\begin{tabular}{|c|c|c|}
\hline $\boldsymbol{\eta}(\mathbf{\%})$ & AFM (eV) & NM (eV) \\
\hline 0 & 0.60 & \\
\hline 7.6 & 0.59 & \\
\hline 13.4 & 0.60 & \\
\hline 20.7 & 0.61 & 0.56 \\
\hline 30.5 & & 0.52 \\
\hline 35.7 & & 0.48 \\
\hline 38.4 & & 0.43 \\
\hline 41.2 & & 0.31 \\
\hline 47.0 & & 0.15 \\
\hline 53.1 & & \\
\hline 56.2 & 0.10 & \\
\hline
\end{tabular}

In addition to the magnetic transformation in the deformed ZGNRs, it is of great importance to examine the electronic properties of the deformed ZGNRs under spin-polarized condition. The variation of the energy gap at the different strain $\eta$ is given in TABEL II. We first consider the case with $\eta=0$, i.e. the flat ZGNR, which presents an energy gap with $0.60 \mathrm{eV}$ and is in good agreement with previous study [13]. Following, ZGNRs with $\eta=7.6 \%, 13.4 \%$ and $20.7 \%$ are calculated, we find the energy gap remains essentially the same, and the system displays the AFM ground state. When $\eta$ $=30.5 \%$, the energy gap undergoes a sudden drop to $0.56 \mathrm{eV}$ and the system becomes a NM semiconductor. High sensitivity of the energy gap upon strain is found. With the elevated strain, the band gap decreases monotonically. For $56.2 \%$-ZGNR, the system exhibits the AFM ground state and has an energy gap of $0.10 \mathrm{eV}$.

\section{CONCLUSIONS}

In summary, we have studied the influence of out-of-plane strain on the electronic properties of ZGNRs using first principles simulations. The results reveal that the electronic properties of ZGNRs are highly sensitive to the different strain. The band gap first remains essentially the same and then decreases monotonically. The diverse electronic properties of ZGNRs can be used to develop and fabricate high sensitive strain sensors.

\section{ACKNOWLEDGMENT}

This work was supported by the National Basic Research Program of China (Grant Nos. 2011CB707601 and 2009CB623702), the National Natural Science Foundation of China (Grant Nos. 51071044, 60976003 and 61006011), Program for New Century Excellent Talents in University (NCEF-09-0293), Specialized Research Fund for the Doctoral Program of Higher Education (20100092110014), and Open Research Fund of State Key Laboratory of Bioelectronics.

\section{REFERENCES}

[1] K. S. Novoselov, A. K. Geim, S. V. Morozov, D. Jiang, Y. Zhang, S. V. Dubonos, et al. "Electric Field Effect in Atomically Thin Carbon Films," Science, vol. 306, pp. 666-669, 2004.

[2] C. Berger, Z. Song, X. Li, X. Wu, N. Brown, C. Naud, et al. "Electronic Confinement and Coherence in Patterned Epitaxial Graphene," Science, vol. 312, pp. 1191-1196, 2006.

[3] A. K. Geim, K. S. Novoselov, "The rise of graphene," Nat. Mater., vol. 6, pp. 183-191, 2007.

[4] T. Ohta, A. Bostwick, T. Seyller, K. Horn, E. Rotenberg, "Controlling the Electronic Structure of Bilayer Graphene," Science, vol. 313, pp. 951-954, 2006.

[5] J. B. Oostinga, H. B. Heersche, X. L. LIU, A. F. Morpurgo, L. M. K. Vandersypen, "Gate-induced insulating state in bilayer graphene devices," Nature Materials, vol. 7, pp. 151-157, 2007.

[6] S. Y. Zhou, G.-H. Gweon, A. V. Fedorov, P. N. First, W. A. De Heer, D.-H. Lee, et al. "Substrate-induced bandgap opening in epitaxial graphene," Nature Materials, vol. 6, pp. 770-775, 2007.

[7] Y. Gan, L. Sun, F. Banhart, "One- and Two-Dimensional Diffusion of Metal Atoms in Graphene,” Small, vol. 4, pp. 587-591, 2008.

[8] O. Cretu, A. V. Krasheninnikov, J. A. Rodriguez-Manzo, L. Sun, R. M. Nieminen, F. Banhart, "Migration and localization of metal atoms on strained graphene," Phys. Rev. Lett., vol. 105, pp. 196102, 2010.

[9] A. V. Krasheninnikov, P. O. Lehtinen, A. S. Foster, P. Pyykko, R. M. Nieminen, "Embedding Transition-Metal Atoms in Graphene: Structure, Bonding, and Magnetism," Phys. Rev. Lett., vol. 102, pp. $126807-$ $126810,2009$.

[10] A. V. Krasheninnikov, R. M. Nieminen, "Attractive interaction between transition-metal atom impurities and vacancies in graphene: a firstprinciples study," Theor. Chem. Acc., vol. 129, pp. 625-630, 2011.

[11] L. Brey, H. A. Fertig, "Electronic states of graphene nanoribbons studied with the Dirac equation," Phys. Rev. B, vol. 73, pp. 235411-235415, 2006 .

[12] Y. W. Son, M. L. Cohen, S. G. Louie, "Energy Gaps in Graphene Nanoribbons," Phys. Rev. Lett., vol. 97, pp. 216803-216806, 2006.

[13] T. B. Martins, R. H. Miwa, A. J. R. da Silva, A. Fazzio, et al. "Electronic and Transport Properties of Boron-Doped Graphene Nanoribbons," Phys. Rev. Lett., vol. 98, pp. 196803-196806, 2007.

[14] F. Cervantes-Sodi, G. Csányi, S. Piscanec, A. C. Ferrari, "Edgefunctionalized and substitutionally doped graphene nanoribbons: Electronic and spin properties," Phys. Rev. B, vol. 77, pp. 165427$165439,2008$.

[15] Y. F. Li, Z. Zhou, P. W. Shen, Z. F. Chen, "Spin Gapless Semiconductor-Metal-Half-Metal Properties in Nitrogen-Doped Zigzag Graphene Nanoribbons," ACS Nano, vol. 3, pp. 1952-1958, 2009.

[16] L. Sun, Q. X. Li, H. Ren, H. B. Su, Q. W. Shi, J. L. Yang, "Strain effect on electronic structures of graphene nanoribbons: A first-principles study," J. Chem. Phys., vol. 129, pp. 074704-074709, 2008.

[17] O. Hod, G. E. Scuseria, "Electromechanical Properties of Suspended Graphene Nanoribbons," Nano Lett., vol. 9, pp. 2619-2622, 2009.

[18] J. Zhang, K. P. Ong, P. Wu, "The Inflence of Out-of-Plane Deformation on the Band Gap of Graphene Nanoribbons," J. Phys. Chem. C, vol. 114 pp. 12749, 2010.

[19] Y. R. Yang, X. H. Yan, X. J. Shen, X. Y. Zhang, Y. Xiao, "Curvature Effects on the Magnetism of Ultrashort Zigzag Carbon Nanotubes and Nanographenes," J. Phys. Chem. C, vol. 114, pp. 7553-7557, 2010.

[20] Z. Z. Yu, L. Z. Sun, C. X. Zhang, J. X. Zhong, "Transport properties of corrugated graphene nanoribbons," Appl. Phys. Lett., vol. 96, pp. 173101-173103, 2010.

[21] Vitor M. Pereira, A. H. Castro Neto, "Tight-binding approach to uniaxial strain in graphene," Phys. Rev. B, vol. 80, pp. 045401-045408, 2009.

[22] M. Farjam, H. Rafii-Tabar, "Comment on "Band structure engineering of graphene by strain: First-principles calculations," Phys. Rev. B, vol. 80, pp. 167401-167403, 2009. 
[23] G. Kresse, J. Furthmüller, "Efficiency of ab-initio total energy calculations for metals and semiconductors using a plane-wave basis set” Comput. Mater. Sci., vol. 6, pp. 15-50, 1996.

[24] G. Kresse, J. Furthmüller, "Efficient iterative schemes for $a b$ initio totalenergy calculations using a plane-wave basis set" Phys. Rev. B, vol. 54, pp. 11169-11186, 1996.

[25] J. P. Perdew, K. Burke, M. Ernzerhof, "Generalized Gradient Approximation Made Simple” Phys. Rev. Lett., vol. 77, pp. 3865-3868, 1996.

[26] P. E. Blöchl, "Projector augmented-wave method" Phys. Rev. B, vol. 50, pp. 17953-17979, 1994.

[27] G. Kresse, D. Joubert, "From ultrasoft pseudopotentials to the projector augmented-wave method" Phys. Rev. B, vol. 59, pp. 1758-1775, 1999.

[28] M. Fujita, K. Wakabayashi, K. Nakada, K. Kusakabe, "Peculiar Localized State at Zigzag Graphite Edge” J. Phys. Soc. Jpn., vol. 65, pp. 1920-1923, 1996.

[29] O. V. Yazyev, "Emergence of magnetism in graphene materials and nanostructures" Rep. Prog. Phys., vol. 73, pp. 056501, 2010.

[30] B. Huang, F. Liu, J. Wu, B.-L. Gu, W. H. Duan, "Suppression of spin polarization in graphene nanoribbons by edge defects and impurities" Phys. Rev. B, vol. 77, pp. 153411-153414, 2008.

[31] W. Z. Wu, Z. H. Zhang, P. Lu, W. L. Guo, "Electronic and magnetic properties of zigzag graphene nanoribbons with periodic protruded edges" Phys. Rev. B, vol. 82, pp. 085425, 2010.

[32] J. Kunstmann, C. Özdoğan, A. Quandt, H. Fehske, "Stability of edge states and edge magnetism in graphene nanoribbons" Phys. Rev. B, vol. 83, pp. 045414-045421, 2010. 